\title{
University of the Philippines - Philippine General Hospital Revised Clinical Practice Guidelines for the Management of Well-Differentiated Thyroid Carcinoma of Follicular Cell Origin
}

\author{
Cherrie Mae Sison ${ }^{1}$, Jerry Obaldo², Jeannette Matsuo ${ }^{3}$, Gemma Leonora Uy ${ }^{4}$, Cristina Jaring 5 \\ on behalf of the UP - PGH Thyroid Cancer Working Group \\ ${ }^{1}$ Clinical Associate Professor, Section of Endocrinology, Diabetes and Metabolism, Department of Medicine, UP - PGH \\ ${ }^{2}$ Associate Professor, Section of Nuclear Medicine, Department of Medicine, UP - PGH \\ ${ }^{3}$ Clinical Associate Professor, Department of Otorhinolaryngology, UP - PGH \\ ${ }^{4}$ Clinical Associate Professor, Department of Surgery, UP - PGH \\ ${ }^{5}$ Fellow, Section of Endocrinology, Diabetes and Metabolism, Department of Medicine, UP - PGH
}

\begin{abstract}
In an effort to unify treatment approaches to patients with well-differentiated thyroid cancer of follicular cell origin, the Section of Endocrinology, Section of Nuclear Medicine, Department of Surgery, and Department of Otorhinolaryngology of the Philippine General Hospital formulated and published a clinical practice guideline on the management of welldifferentiated thyroid cancer in 2008. In recognition of the new literature on thyroid cancer, as well as the questions that have arisen from the use of the guideline, the group reconvened in 2011 to review and update the clinical practice guideline designed for patients seen at the Philippine General Hospital. This article summarizes the latest revisions and recommendations.
\end{abstract}

Keywords: Thyroid cancer, clinical practice guideline, UP - PGH

\section{Contents}

I) Introduction
A) Background
B) Objectives
C) Methodology
D) Levels of evidence and recommendation

II) Summary of Issues and Revisions

III) Definition of Terms

IV) Recommendations

A) Primary Therapy

1) Surgical approach

2) Preoperative cervical ultrasound

3) Frozen section

4) Lymph node dissection

5) Completion thyroidectomy

B) Disease Staging

C) Adjunctive Therapy

1) Radioactive iodine ablative therapy

2) TSH suppression therapy

3) Radiotherapy

4) Chemotherapy

5) Novel therapies

D) Follow-up and Monitoring
1) Thyroglobulin
2) Anti-thyroglobulin antibody
3) Whole body I-131 scan
4) Cervical ultrasound

ISSN 0857-1074

Printed in the Philippines

Copyright $\odot 2012$ by the JAFES

Received March 26, 2012. Accepted April 11, 2012.

\section{I) Introduction}

The University of the Philippines - Philippine General Hospital (UP - PGH) is a government-assisted, tertiary, teaching hospital catering to patients from all over the Philippines. Most of the patients are from the lower socioeconomic classes, putting agreat strain on the hospital's resources. Financial constraints notwithstanding, the institution is the home of many leading local experts from various specialties, who, together with their trainees and allied medical staff, are committed to giving the best available healthcare possible.

Adult patients with well-differentiated thyroid cancer are seen through 3 portals of entry: Department of Surgery, Department of Otorhinolaryngology and Section of Endocrinology of the Department of Medicine. Annually there are about 88 to 330 new cases of thyroid cancer seen at the UP-PGH (census data on file). Although collaborations between medical and surgical departments are usual, no unifying treatment protocol is used.

In an endeavor to standardize and optimize treatment offered to adult patients with well-differentiated thyroid cancer, endocrinologists, surgeons, otorhinolaryngologists, and nuclear medicine physicians at the UP-PGH met 
together in 2005 to formulate a practice guideline. After a long process of formulating objectives, presenting respective section protocols, identifying issues, retrieving and reviewing evidence, and finally trying to arrive at a consensus, a clinical practice guideline (CPG) for the management of UP - PGH patients with thyroid nodules and well differentiated thyroid cancer has been drafted and subsequently published in $2008 .^{1}$

Since then new literature on the management of thyroid nodules and thyroid cancer has proliferated. Contemporary issues regarding the applicability of some of the recommendations have also been raised. Hence, in 2011 the interdisciplinary panel of experts responsible for the UP-PGH CPG for thyroid nodules and welldifferentiated thyroid cancer reconvened with three objectives in mind: 1) Present the experiences and collected data using the current recommendations; 2) Review the appropriateness of the recommendations given the current institutional milieu; and 3) Update the CPG as deemed necessary. The same methodology in reviewing evidence and arriving at a consensus has been carried out. ${ }^{2}$ Grading of the levels of evidence and strength of recommendation are similar (see Tables 1 and 2). Following monthly discussions from September 2011 to January 2012, the UP-PGH Working Group on Thyroid Cancer has completed this revised CPG.

\section{Table 1. Levels of Evidence}

Evidence form at least one properly designed randomized, controlled trial or meta-analysis

II - Evidence from at least one well-designed clinical trial without prior proper randomization, from prospective cohort or casecontrol analytic studies (preferably from one center), from multiple time-series studies, or from dramatic results in uncontrolled experiments

III - Evidence from opinions of respected authorities on the basis of clinical experiences, descriptive studies, or reports of expert committees

\section{Table 2. Categories of Recommendations}

A - Recommendations that were approved by consensus (75\% of the multi-sectoral expert panel).

Strongly recommends (Homogenous evidence from multiple well-designed randomized controlled trials with sufficient statistical power; homogenous evidence from multiple well-designed cohort controlled trials with sufficient statistical power; at least one conclusive level 1 publication demonstrating benefit outweighs risk)

B - Recommendations that were somewhat controversial and did not meet consensus

Recommends (Evidence from at least 1 well designed clinical trial, cohort, or case-controlled analytic study or metaanalysis; non-conclusive level 1 publication; at least one conclusive level 2 publications demonstrating benefit outweighs risk)

C - Recommendations that caused real disagreements among members of panel

Recommendation is based on expert opinion (Evidence based on clinical experience, descriptive studies, or expert consensus opinion; no conclusive level 1 or 2 publication; at least 1 conclusive level 3 publication demonstrating benefit outweighs the risk)
The working group would like to reiterate that this CPG is tailored to fit the qualities of adult, nonpregnant Filipino patients seen at UP - PGH, as well as to the expertise and other material resources available in the institution. It is by no means a national guideline. Applicability to other institutions or healthcare providers will be left to the judgment of the clinician.

\section{II) Summary of issues and revisions}

Several questions were raised during the monthly discussions. Issues include the applicability of the guideline to all thyroid nodules, clarification of definition of terms, the primary and adjunctive therapy, and on the post-treatment follow up. Below is a summary of the consensus on the issues raised.

A) Should the guideline for the evaluation of the thyroid nodule be separated from the guideline for well-differentiated cancer?

The consensus is to separate the guideline for the management of thyroid nodules from that of the management of well-differentiated cancers. The panel agrees that this will focus discussions and minimize controversies. Users of the CPG will also find the resulting manuscript easier to digest.

B) Are there any other terminologies we wish to clarify?

POST-THERAPY SCAN: Scan performed using the therapeutic dose of I-131. It is usually performed within 2 weeks after dose administration

PRE-THERAPY OR "DIAGNOSTIC" SCAN: Scan performed $1-7$ days after a diagnostic dose of $2-5 \mathrm{mCi}(74-185 \mathrm{MBq})$ of I-131.

C) Should we risk stratify patients prior to recommending a primary surgical approach?

The consensus is to maintain the original recommendation of total or near total thyroidectomy for all patients with welldifferentiated thyroid cancer and a nodule size greater than $1 \mathrm{~cm}$ in largest diameter. Thyroid lobectomy may be sufficient treatment for thyroid cancers $1 \mathrm{~cm}$ or less in diameter, isolated, intrathyroidal, and without cervical lymph node metastasis on preoperative ultrasound (Grade B)

D) Is preoperative cervical ultrasound recommended? A preoperative cervical ultrasound is recommended for all adult patients with welldifferentiated thyroid cancer. (Grade A)

E) What is the role of intraoperative frozen section? A frozen section may be performed for nondiagnostic preoperative FNAB result. (Grade B) 
F) What type of lymph node dissection should be performed?

An appropriate therapeutic lymph node dissection shall be performed if clinical and radiologic evaluations show evidence of nodal involvement. Prophylactic node dissection is not recommended at this time. (Grade A)

G) When should completion thyroidectomy be performed?

Patients with well-differentiated cancer who underwent lobectomy as initial surgery shall be offered completion thyroidectomy if at least 1 of the following is present: nodal metastasis, gross residual tumor, extra-thryoidal extension, vascular invasion, aggressive histopathology, nodules in the contralateral lobe. (Grade A)

H) Is repeat radioactive iodine therapy warranted? Repeat RAI therapy may be performed while there is detectable thyroid tissue remnant or metastasis. (Grade A)

I) Should anti-thyroglobulin antibody be used as an indicator for the need for subsequent RAI therapy?

Although the panel agrees that the use of elevated serum anti-thyroglobulin is promising as an indicator for RAI therapy, existing foreign and local data is insufficient to recommend its use. (Grade A)

J) What activity of I-131 should be used for remnant ablation?

An I-131 dose of 30 - 100 mCi (1,110 - 3,700 MBq) shall be given to patients with no nodal extension, perithyroidal extension, or distant metastases. (Grade A)

K) What is the role of recombinant human TSH (rhTSH)?

Patients who are unable to tolerate hypothyroidism and/or unable to generate an endogenous elevation of the TSH may be given rhTSH in preparation for diagnostic scanning or RAI therapy. (Grade A)

L) To what level should TSH be suppressed with levothyroxine in low risk patients or in high risk patients at risk for developing complications from thyroid hormone therapy?

Maintenance of TSH at or slightly below the lower limit of normal $(0.1-0.27 \mathrm{mU} / \mathrm{L})$ is appropriate. (Grade A)
M) What is the role of external beam radiation therapy?

Among the indications are tumors that do not concentrate RAI (Grade A)

N) What is the role of novel therapies such as tyrosine kinase inhibitors?

Current evidence is insufficient to recommend their use outside of a research setting. (Grade A)

O) What is the role of postoperative follow up?

Follow up and monitoring is performed for the purpose of determining the need for repeat RAI therapy or other therapeutic modalities. (Grade A)

P) Should whole body ${ }^{131}$ I scintigraphy be performed if there is negative stimulated thyroglobulin levels, anti-thyroglobulin levels, and cervical ultrasound?

Whole body scan may be performed if persistent disease is suspected, despite a negative finding in the other tests. (Grade C)

\section{III) Definition of Terms}

A) Nondiagnostic biopsy: Fail to meet specified criteria for adequacy that have previously been established.

B) Indeterminate cytology: Reported as "suspicious," "follicular lesion," "follicular neoplasm," or "Hurthle cell neoplasm."

C) Total thyroidectomy: Removal of all grossly visible thyroid tissues

D) Near total thyroidectomy: Leave less than 1 gram of tissue adjacent to the insertion of the recurrent laryngeal nerve into the cricothyroid muscle

E) Subtotal thyroidectomy: Leave more than 1 gram of tissue with the posterior capsule on the involved side

F) Absence of persistent tumor: In a patient who has undergone total or near total thyroidectomy and thyroid remnant ablation with radioactive iodine, disease free status comprises all of the following:

1) No clinical evidence of tumor

2) No imaging evidence of tumor (No tumor uptake outside the thyroid bed on the initial posttreatment whole body I131 scan, recent diagnostic scan or neck ultrasound)

3) Undetectable serum thyroglobulin levels during TSH suppression and stimulation, in the absence of interfering antibodies

F) Aggressive histopathologic subtypes: tall cell, columnar cell, insular carcinoma

G) Post-therapy scan: Scan performed using the therapeutic activity of I-131. It is usually 
performed within 2 weeks after dose administration

H) Pre-therapy / "Diagnostic" scan: Performed 1 to 7 days after a diagnostic activity ranging from 2 to $5 \mathrm{mCi}$ (74 to $185 \mathrm{MBq}$ ) of I-131.

\section{IV) Recommendations}

\section{A) Primary Therapy}

1) What is the appropriate extent of initial surgery for differentiated thyroid cancer?

Total or near total thyroidectomy is
recommended for all patients with well-
differentiated thyroid cancer and a nodule
size greater than $1 \mathrm{~cm}$ in largest diameter.
Thyroid lobectomy may be sufficient
treatment for thyroid cancers $1 \mathrm{~cm}$ or less in
diameter, isolated, intrathyroidal, and
without cervical lymph node metastasis on
preoperative ultrasound (Grade B)

It is accepted that adequate surgery remains the key to the successful management of well-differentiated cancers. However, the definition of "adequate surgery" remains controversial. Previous international guidelines tout that the critical tumor size is $>4 \mathrm{~cm}$. This is supported by research from the Memorial Sloan-Kettering Cancer Center showing that low risk patients (i.e., age less than 45 years, tumor size $<4 \mathrm{~cm}$, papillary in histology, absent distant metastasis) do well with a lobectomy. ${ }^{2}$ However, that center is known to have an excellent postoperative follow up of patients. In contrast, many of the patients in UP - PGH are lost to follow up for various reasons. Hence, a less aggressive primary surgical approach, particularly for tumors $<4 \mathrm{~cm}$ in size, may not be appropriate. In addition, there is good evidence for doing an aggressive initial surgical approach. Several studies show that increased extent of primary surgery may improve survival for high risk patients,,$^{3-7}$ and decrease recurrence rates for low risk patients. ${ }^{6-8}$ Multivariate analysis of data from over 50,000 patients with papillary thyroid cancer show that total thyroidectomy significantly improved recurrence and survival rates for tumors $>1.0 \mathrm{~cm}$, even for low risk patients. The study also notes that tumor size $1-2 \mathrm{~cm}$ had $24 \%$ higher risk of recurrence and $49 \%$ higher mortality. ${ }^{9}$

Many guidelines also recommend preoperative risk stratification prior to applying a certain surgical approach. In the
2006 Asia Pacific Region Expert Consensus, ${ }^{10}$ bilateral total or near-total thyroidectomy is recommended when papillary, follicular, or Hurthle cell cancer is known or suspected preoperatively. Additional criteria for preoperative total or near total thyroidectomy were: age greater than 45 years old, tumor more than $2 \mathrm{~cm}$ in diameter, extra thyroidal extension, regional lymph node involvement, multicentricity, history of neck irradiation, family history of cancer, and known distant metastases. For apparently low-risk patients, individualized clinical judgment is advised in light of the varying surgical expertise, minimally lower risk of disease recurrence, and local rates of postoperative hypoparathyroidism and recurrent laryngeal nerve injury.

Nevertheless, the study by Racho and Abrahan suggests that a more aggressive initial approach produces better outcomes even in low-risk patients. This 10-year follow up of 132 patients with stage I or stage II papillary thyroid cancer seen at the Thyroid Clinic of UP-PGH from January 1986 to December 1990 reports greater occurrence of recurrences ( $28 \%$ versus $0 \%$ ) and metastasis $(61.7 \%$ versus $17.6 \%, \mathrm{p}<0.01)$ in patients who underwent non-total thyroidectomy compared to those who had total thyroidectomy plus radioactive iodine ablation. No association is noted between the presence of metastasis and the sex, clinical stage, or family history. ${ }^{11}$ It is unclear whether the better outcomes are due to the extent of the primary surgery or the addition of radioactive iodine ablation. Hence, in the consensus meeting, the panel of experts has agreed to defer preoperative risk stratification in favor of a more aggressive primary approach of total or near total thyroidectomy for all thyroid cancers $>1 \mathrm{~cm}$ in size. Whether such an approach will lead to better outcomes will be the subject of a future review of this clinical practice guideline. Moreover, representatives from the Department of Surgery and Otorhinolaryngology claim to have low rates of postoperative complications with total and near-total thyroidectomy. In the 1998 study of Racho and Abrahan, a 44\% surgeryrelated complication rate is noted. However, there is no significant difference in complication rates between total and nontotal thyroidectomy ( $45 \%$ versus $42 \%$, $\mathrm{p}=0.148) .{ }^{11}$ In a more recent review, the Department of Otorhinolaryngology reports 
no morbidity for the twenty cases of thyroid cancer operated on during the first quarter of 2012 (Data on file).

2) Is preoperative cervical ultrasound recommended?

A preoperative cervical ultrasound is recommended for all adult patients with well-differentiated thyroid cancer. (Grade A)

Cervical lymph node involvement of papillary thyroid carcinoma is found in 20 $50 \%$ of patients. ${ }^{12-16}$ Node metastasis may be present even when the primary tumor is small and intrathyroidal. ${ }^{17}$ Preoperative ultrasound can identify suspicious cervical nodes in $20-31 \%$ of patients ${ }^{18-19}$ and potentially alter the surgical approach in $20 \%$ of cases. ${ }^{20-21}$ Sonographic features suggestive of abnormal metastatic lymph nodes include loss of fatty hilus, rounded rather than oval shape, hypoechogenicity, cystic change, calcifications, and peripheral vascularity. ${ }^{22}$ This modality and the expertise that it requires is readily available in our institution.

3) What is the role of intraoperative frozen section?

A frozen section may be performed for nondiagnostic preoperative fine needle aspiration biopsy results. (Grade B)

Results from studies in the Asia-Pacific region show no consistent agreement between frozen section (FS) and final histopathologic findings. ${ }^{10}$ This experience is paralleled in our institution. Most international guidelines do not recommend its use or ascribe a very limited role to it. A high frequency of false negative results for follicular neoplasia has been noted. ${ }^{23}$ In a retrospective review of 215 patients treated by the same surgeon, the sensitivity and specificity of FS are $32.4 \%$ and $96.5 \%$, respectively. The inter-test agreement between preoperative fine needle aspiration cytology (FNAC) and FS is poor (kappa = 0.17). Frozen section result does not influence treatment decisions when preoperative FNAC is already malignant. On the contrary, on the background of a benign, indeterminate, or nondiagnostic FNAC finding, a malignant FS result influences treatment decisions in $88 \%$ of cases. ${ }^{24}$ In another study involving 210 patients, FS is found most useful for cases diagnosed by preoperative FNAB as suspicious for papillary thyroid cancer. ${ }^{25}$

4) What type of lymph node dissection will be performed?

An appropriate therapeutic lymph node dissection shall be performed if clinical and radiologic evaluations show evidence of nodal involvement. Prophylactic node dissection is not recommended at this time. (Grade A)

Regional lymph node metastases are present in $20-90 \%$ of patients with papillary thyroid cancer (PTC). ${ }^{13,21}$ In the Surveillance, Epidemiology, and End Results (SEER) database $^{25}$, among 9904 patients with PTC, lymph node metastasis is among the factors that predicted a poor outcome. All-cause survival at 14 years was $82 \%$ for PTC without lymph node and 79\% with lymph node metastases $(p<0.05)$. A more recent SEER registry notes that the risk for decreased survival with lymph node metastasis is found only in patients with follicular cancer and those with papillary cancer over age 45 years. ${ }^{27}$ Moreover, the risk for regional recurrence is higher in patients with lymph node metastases. ${ }^{28}$

Bilateral central node dissection may improve survival compared to historical controls and reduce risk for nodal recurrence. ${ }^{29-30}$ Central compartment dissection can be done with low morbidity by experienced surgeons. ${ }^{31-34}$ In the AsiaPacific region selective central neck dissection is usually performed at initial surgery, while lateral neck dissections are reserved for cases in which additional disease is detected either pre- or intraoperatively. ${ }^{10}$ In our institution, both surgeons and otorhinolaryngologists perform selective neck dissection for lymph nodes at levels II - VI only if the patient has clinically positive cervical lymph nodes.

5) When will completion thyroidectomy be performed?

Patients with well-differentiated cancer who underwent lobectomy as initial surgery shall be offered completion thyroidectomy if at least 1 of the following is present: $>1 \mathrm{~cm}$ original tumor size, multifocal disease, nodal metastasis, gross residual tumor, extrathyroidal extension, vascular invasion, aggressive histopathology, nodules in the contralateral lobe. (Grade A) 
Completion thyroidectomy allows for complete resection of a multicentric disease, ${ }^{35}$ improved efficacy of postoperative ablative radioactive iodine (RAI) therapy, and simpler postoperative monitoring for disease persistence or recurrence. The surgical risks of a two-stage thyroidectomy have been shown to be similar to those of a near total or total thyroidectomy. ${ }^{36-37}$ In the 2006 report from the Asia-Pacific region, completion thyroidectomy is advised for patients with large tumor size, local extrathyroidal invasion, involved resection margins, extensive cervical node metastases, aggressive histology, multifocal tumors, vascular invasion, and nodules in the contralateral remaining lobe. These characteristics are believed to confer an increased risk of disease recurrence. In addition, experts prefer surgical excision of the remaining tissue to the multiple I-131 doses typically required to ablate an entire remaining lobe. ${ }^{10}$

\section{B) Disease Staging}

1) What postoperative staging system will be used?

The $6^{\text {th }}$ edition of the American Joint Committee on Cancer/International Union Against Cancer (AJCC/UICC) TNM classification shall be used for all patients with differentiated thyroid cancer because of its utility in predicting disease mortality and its requirement for cancer registries. (Grade A)

Post-op staging is used for prognostication, decision making on adjunctive therapy, to determine frequency and intensity of follow up, and to enable accurate communication between health care professionals. No scheme has demonstrated clear superiority. ${ }^{38}$ Each of the schemes allows accurate identification of $70-85 \%$ of patients at low risk of mortality. The AJCC/UICC TNM staging employs a shorthand method and is used for hospital cancer registries and epidemiologic studies.

2) What risk stratification will be used to assess the risk of cancer recurrence?

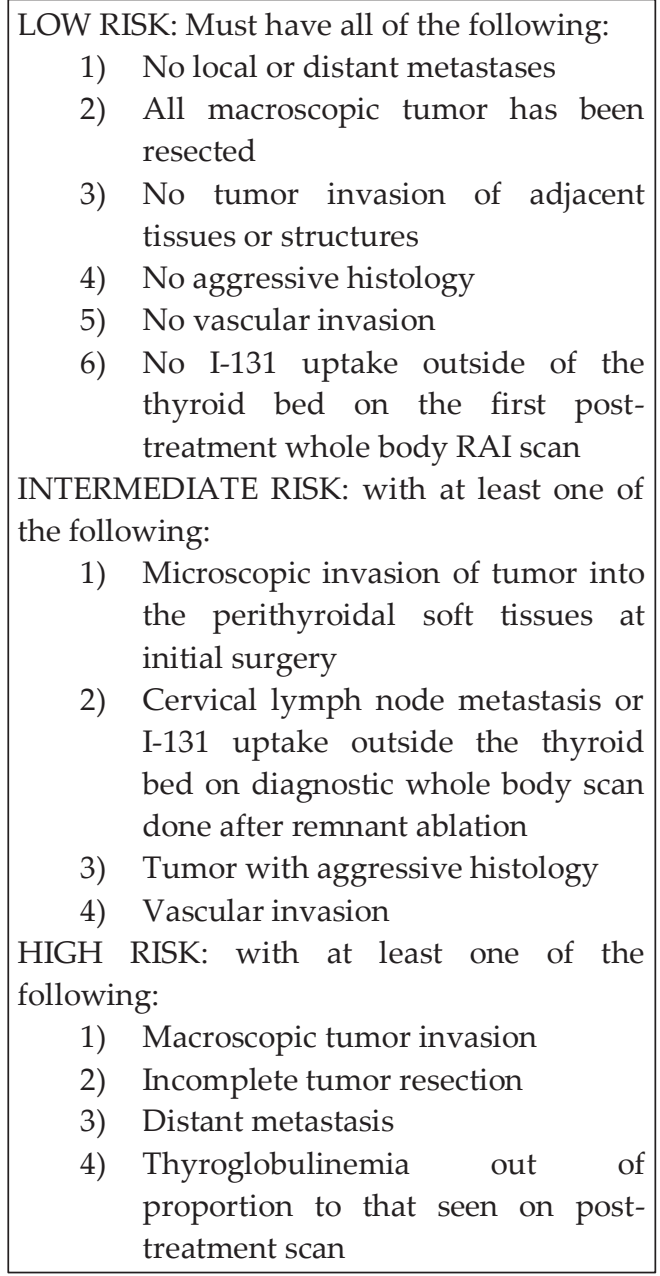

The AJCC/IUCC staging is designed to predict death and not recurrence. Hence, the above risk stratification is adopted from the 2009 American Thyroid Association Guidelines. ${ }^{22}$

\section{C) Adjunctive Therapy}

\section{1) Radioactive Iodine Therapy}

a) Who are candidates for radioactive iodine (RAI) therapy?

Adjunctive ablative radioactive iodine-131 therapy will be given postoperatively to all patients with well-differentiated thyroid carcinoma assessed to be at INTERMEDIATE TO HIGH RISK for disease morbidity and mortality. Repeat RAI therapy may be performed while there is detectable thyroid tissue remnant or metastasis. (Grade A)

The purposes of postoperative RAI therapy are to 1) achieve remnant ablation to facilitate detection of recurrent disease and initial staging; 2) provide adjuvant therapy to decrease the risk of recurrence and diseasespecific mortality; or 3) act as RAI therapy to treat known persistent disease. ${ }^{22}$ 
Postoperative radioiodine remnant ablation destroys residual thyroid tissue to facilitate long-term surveillance with whole-body iodine scans and/or stimulated $\mathrm{Tg}$ measurements. ${ }^{6,39}$ Large, retrospective trials show significant decrease in disease recurrence $^{40-44}$ and cause-specific mortality with adjuvant RAI treatment. ${ }^{4-5,40-42}$ Benefit appears to be restricted to those with tumor size greater $1.5 \mathrm{~cm}$ or with residual disease after surgery. Lower risk patients do not show evidence for benefit. $4,6,44 \quad$ No prospective studies have been performed to address this problem. Hence, in the AsiaPacific expert consensus, some low risk patients may not receive radioiodine, particularly if the prospects for reliable longterm follow up are good. ${ }^{10}$

\section{b) What activity of I-131 will be used for remnant ablation?}

An I-131 activity of $30-100 \mathrm{mCi}(1,110-$ 3,700 MBq) will be given to patients with no nodal extension, perithyroidal extension, or distant metastases. An activity of $150 \mathrm{mCi}$ (5,550 MBq) shall be given to patients with nodal metastases and to patients with distant metastases on their first radioiodine therapy. Subsequent radioiodine ablation of patients with distant metastases will use $200 \mathrm{mCi}$ $(7,400 \mathrm{MBq})$, except in the presence of diffuse lung metastases where $150 \mathrm{mCi}(5,550 \mathrm{MBq})$ is recommended. (Grade A)

Successful remnant ablation, usually defined as absence of visible radioiodine uptake on a subsequent diagnostic radioiodine scan, is generally achieved with activities of I-131 between 30 and $100 \mathrm{mCi}^{57-60}$ However, higher activities tend to have higher success rates. ${ }^{62}$ An activity of $100-200 \mathrm{mCi}$ may be appropriate if residual macroscopic disease is suspected or documented or if there is an aggressive tumor histology. In the 2006 AsiaPacific report, ${ }^{10}$ I-131 activities used for therapy typically range from $50-150 \mathrm{mCi}$. A recent retrospective review (data on file) looks at differences in ablation rates and adverse effects between 50 and $100 \mathrm{mCi}$ I-131 activities. In 74 adults who underwent radioactive iodine therapy after total thyroidectomy in Saint Luke's Medical Center for low stage well-differentiated thyroid cancer, successful remnant ablation $(66.7 \%$ versus $76 \%)$ is comparable between the two I-131 activities. Administration of 50 $\mathrm{mCi}$ is associated with fewer short-term adverse effects in this study. Unfortunately, the paucity of local studies precludes the panel from clearly identifying which I-131 activity is the most cost-effective. Hence, the actual dose to be given to a patient will still depend on the judgment of the clinician.

c) How will patients be prepared for radioiodine ablation?

Post-op radioactive iodine therapy will be given within $4-6$ weeks after the surgery. Low iodine diet will be prescribed. Levothyroxine will not be given prior to radioactive iodine therapy. For subsequent radioactive iodine therapy, levothyroxine therapy will be withdrawn for at least three weeks prior to the time of therapy. (Grade A)

Remnant ablation requires TSH stimulation. Uncontrolled studies suggest that TSH $>30$ $\mathrm{mU} / \mathrm{L}$ is associated with increased RAI uptake in tumors. ${ }^{47}$ In our institution, since liothyronine is unavailable, endogenous TSH elevation is usually achieved by withdrawing levothyroxine for 3 - 4 weeks. This approach has been shown to achieve $\mathrm{TSH}>30 \mathrm{mU} / \mathrm{L}$ in more than $90 \%$ of patients. ${ }^{49-52}$

d) Is there a need for pre-therapy whole body scintigraphy with I-131?

A pre-therapy whole body I-131 scan is not recommended due to potential problems with detection sensitivity and post-imaging stunning, (Grade A)

In the presence of a large thyroid remnant, the whole body I-131 scan is dominated by uptake within the remnant, potentially masking the presence of extrathyroidal disease within locoregional lymph nodes, the upper mediastinum, or even at distant sites. ${ }^{44}$ In addition, there is an increasing trend to avoid pre-therapy radioiodine scans due to concerns over I-131 induced stunning of the thyroid cancer. ${ }^{45}$ Stunning will produce a reduction in the uptake of the I-131 therapy activity. This occurs most prominently with higher I-131 activities (5-10 $\mathrm{mCi}$ ) and with increasing time between the diagnostic dose and therapy. ${ }^{46-47}$ Although stunning is not visually appreciated at activities of $1-3 \mathrm{mCi}$, the accuracy of low dose scans has been questioned, particularly in patients who have had previous RAI therapy. Furthermore, even if studies have shown excellent concordance between I-123 and I131 for tumor detection, routine use of I-123 is not recommended for the following 
reasons: 1) optimal I-123 activity and time to scan after I-123 administration is not known; 2) I-123 is expensive and not universally available; 3 ) the short half-life ( $\mathrm{t}_{1 / 2}=13$ hours) of I-123 makes handling this isotope more difficult; 4) stunning may also occur, although to a lesser degree than I-131.47 Hence, in the 2006 Asia-Pacific report of experts, ${ }^{10}$ pre-therapy diagnostic scan is typically not performed Instead, a posttherapy scan is usually obtained to define the amount and site(s) of remaining thyroid tissue.

e) What is the role of recombinant human TSH (rhTSH)?

Recombinant human TSH may be given to
patients with contraindications to
levothyroxine withdrawal and/or who are
unable to endogenously raise their TSH
levels. (Grade A)

The inconvenience of symptomatic hypothyroidism and the theoretical probability that prolonged periods of hyperthyrotropinemia could stimulate cancer cell growth have prompted strategies aimed at reducing or eliminating the time these patients must spend without thyroid hormone. Use of recombinant human TSH is probably the best solution to avoid hypothyroidism. Studies have found thyroid hormone withdrawal and rhTSH approaches to be equally effective in preparing patients for remnant ablation ${ }^{55-56}$ or diagnostic examination. However, it is expensive and not readily available in our institution.

\section{2) What is the role of TSH suppression therapy?}

Initial TSH suppression to below $0.1 \mathrm{mU} / \mathrm{L}$ is recommended for high risk patients in the absence of contraindications such as decreased bone mineral density, atrial fibrillation, or myocardial ischemia. Maintenance of TSH at or slightly below the lower limit of normal (0.1 to $0.27 \mathrm{mU} / \mathrm{L}$ ) is appropriate for low to intermediate risk patients and for high-risk patients at risk for developing complications from thyroid hormone suppressive therapy. (Grade A)

The goals of thyroid hormone therapy are to restore euthyroidism and suppress circulating TSH to reduce the risk of tumor recurrence. A meta-analysis has suggested an association between thyroid hormone suppression therapy and reduction of major adverse clinical events. ${ }^{62}$ There is a study that showed a $\mathrm{TSH} \leq 0.05 \mathrm{mU} / \mathrm{L}$ is associated with longer relapse-free survival, ${ }^{63}$ while another large study did not show correlation between disease progression and TSH suppression. ${ }^{64}$ On the other hand, the potential benefit from thyroid hormone suppression should be weighed against the risk of complications from such treatment. There is a strong association with the development of atrial fibrillation when the TSH is less than $0.10 \mathrm{mU} / \mathrm{L}$, whereas the evidence for risk of atrial fibrillation with a serum TSH of 0.1 to $0.3 \mathrm{mU} / \mathrm{L}$ is limited. ${ }^{65}$ The risk for atrial fibrillation can be anywhere from 2.8 fold over 2 years or 3 fold in 10 years. ${ }^{66-67}$ The strength of association with cardiac dysfunction and reduced bone mineral density in postmenopausal women is fair. ${ }^{65}$

The appropriate degree of TSH suppression by levothyroxine is still unknown. In the 2006 Asia-Pacific report, ${ }^{10}$ the precise intensity of TSH suppression depends on the patient's disease stage and the sensitivity of available TSH assays. Some clinicians reduce the intensity of TSH suppression after several years in patients with no evident disease, particularly if there are symptoms of thyrotoxicosis, declining bone mineral density, risk of atrial fibrillation or myocardial ischemia. It is likewise advisable to reduce the intensity of TSH suppression in low risk patients with no recurrent disease 2 years postoperatively. However, in high-risk patients and in those with residual disease, beta blockers may be necessary to permit the adequate TSH suppression. ${ }^{22}$

3) What is the role of external beam radiation therapy?

External beam radiation therapy shall be reserved for the management of the following situations: unresectable gross residual cervical disease that does not concentrate RAI, painful bone metastases, metastatic lesions in critical locations likely to result in fracture, neurological or compressive symptoms not amenable to surgery, painful pleural based lesions, and recurrent hemoptysis. (Grade A)

Due to the efficacy of radioactive I-131 therapy, external beam radiation is infrequently used in the management of well-differentiated thyroid cancer. Its role is limited to palliation of unresectable disease. ${ }^{68}$ Furthermore, it is not known whether 
external beam radiation might reduce the risk for recurrence in the neck after adequate primary surgery and/or radioactive iodine therapy.

\section{4) What is the role of chemotherapy?}

The routine adjunctive use of chemotherapy is not recommended. However, it may be considered in patients who have surgically unresectable disease and are unresponsive to radioactive $\mathrm{I}^{131}$ or external beam radiation therapy. It may also be offered to patients who are not amenable to external beam radiotherapy. (Grade $\mathrm{A}$ )

Doxorubicin monotherapy may produce partial response or stable disease in up to $40 \%$ of patients, ${ }^{69-72}$ but durable responses are uncommon. Most studies of combination chemotherapy show no advantage over monotherapy. ${ }^{73}$

5) What is the role of novel therapies such as tyrosine kinase inhibitors?

Current evidence is insufficient to recommend their use outside of a research setting. (Grade A)

A minority of patients with welldifferentiated thyroid cancer will experience progressive, life-threatening growth and spread of the disease. A variety of targeted therapies are now under clinical evaluation in phase I to III studies. Among the agents in focus are tyrosine kinase inhibitors, PPAR $\gamma$ activators, vascular endothelial growth factor receptor inhibitors, immunomodulators, and p53 tumor suppressor gene activators. Results are promising but none of these drugs have yet been specifically approved for the treatment of metastatic thyroid carcinoma.

\section{D) Post-operative Follow up and monitoring}

\section{1) What is the role of postoperative follow up?}

Follow up and monitoring is performed for the purpose of determining the need for repeat RAI therapy or other therapeutic modality. (Grade A)

A major goal of long-term follow up is accurate surveillance for possible recurrence. It is believed that early detection of recurrent disease offers the best opportunity for effective treatment. Another goal of postoperative follow up is to monitor the adequacy of thyroxine suppression or replacement therapy. ${ }^{22}$

2) What is the role of thyroglobulin measurements?

In patients with well-differentiated thyroid cancer who have undergone total or neartotal thyroidectomy and postoperative radioiodine ablation, TSH stimulated serum thyroglobulin should be measured every 6 12 months by an immunometric assay, ideally in the same laboratory and using the same assay. Concurrent determination of thyroglobulin antibodies should be done at least once during follow up to determine the reliability of the thyroglobulin levels obtained. A basal and stimulated thyroglobulin level of $\leq 1 \mathrm{ng} / \mathrm{ml}$ and $\leq 2$ $\mathrm{ng} / \mathrm{ml}$, respectively, are indicative of absence of persistent disease. (Grade A)

Routine serum thyroglobulin determination is not recommended in patients with less than total thyroidectomy and in patients with total thyroidectomy but no ablative radioactive iodine treatment. (Grade $\mathrm{A}$ )

Serum thyroglobulin has a high sensitivity and specificity in detecting thyroid cancer, especially after total thyroidectomy and remnant ablation with I-131. The highest degree of sensitivity is noted when a TSH concentration of $25-30 \mathrm{mU} / 1$ or more has been reached, commonly after thyroid hormone withdrawal for at least 3 weeks. ${ }^{74-77}$ Although thyroglobulin determination may be done during concurrent thyroid hormone suppression, a negative result (i.e., less than $1 \mathrm{ng} / \mathrm{mL}$ ) will necessitate repeating the test under TSH stimulation to avoid missing patients with relatively small amounts of residual tumor. ${ }^{78-79}$ Patients not amenable to or with contraindications to thyroid hormone withdrawal may use recombinant human TSH. According to the panel of experts in the Asia-Pacific report, ${ }^{10}$ TSHstimulated serum thyroglobulin testing alone, without whole-body scanning, may be sufficiently sensitive to monitor thyroid cancer patients who have previously had a negative radioiodine scan. This approach is recognized to be more cost-effective and convenient in settings where whole body I131 scintigraphy is logistically difficult and expensive. 
Thyroglobulin antibodies found in $25 \%$ of thyroid cancer patients ${ }^{80}$ will falsely lower serum thyroglobulin measurements in immunometric assays. $^{81}$ Less commonly, defective or absent production of immunoreactive thyroglobulin by tumor cells may fail to identify patients with clinically significant tumors, even when thyroglobulin determination is done under TSH stimulation. ${ }^{82}$ Small cervical lymph node metastases and less differentiated tumors are other sources of false negative thyroglobulin results. ${ }^{83-84}$

3) Should anti-thyroglobulin antibody be used as an indicator for the need for subsequent RAI therapy?

Although the panel agrees that the use of elevated serum anti-thyroglobulin is promising as an indicator for RAI therapy, existing foreign and local data is insufficient to recommend its use. (Grade A)

There are studies that suggest serial serum antithyroglobulin antibody measurements have independent clinical value for following well-differentiated thyroid cancers with thyroglobulin antibody positivity. It may serve as an imprecise surrogate marker of residual thyroid tissue. ${ }^{85-86}$ Retention of antithyroglobulin antibody positivity during long term follow-up indicates persistent disease. ${ }^{85}$ The disappearance of TgAbs after therapy seems to represent a favorable prognostic factor. ${ }^{87}$ However, cut-off points which will indicate the need for RAI therapy remain unclear from the current literature.

\section{4) Whole Body I-131 scintigraphy}

i) What is the role of whole body I-131 scintigraphy?

A post-therapy whole body I-131 scan will be done within 3 to 7 days in all patients who have undergone ablative radioactive iodine therapy. Thereafter, patients with negative stimulated thyroglobulin levels, antithyroglobulin levels and cervical ultrasound do not require routine diagnostic whole body scan during follow up. (Grade A)

A diagnostic whole body I-131 scan is most useful during follow up when there is little or no remaining thyroid tissue. Stunning and accuracy are two issues that have to be addressed. Low risk patients who are clinically free of residual tumor, have undetectable serum thyroglobulin levels, and negative cervical ultrasound usually do not require diagnostic pretreatment scans. On the other hand, the posttreatment scan, due to the larger doses of I-131 used, may occasionally visualize disease not seen on pretreatment scan. ${ }^{89-93}$ Historically, it has been the practice in many centers in the AsiaPacific region to do radioiodine scanning every $6-12$ months until two sequential negative scans are obtained. ${ }^{22}$ With wider implementation of basal and TSH-stimulated thyroglobulin monitoring, a single negative whole-body scan is now considered sufficient to cease further scanning unless thyroglobulin levels are rising or are unreliable due to the presence of autoantibodies.

ii) Should whole body I-131 scintigraphy be performed if there is negative stimulated thyroglobulin levels, anti-thyroglobulin levels, and cervical ultrasound?

Whole body scan may be performed if persistent disease is suspected, despite a negative finding in the other tests. (Grade C)

\section{5) What is the role of cervical ultrasound?}

Cervical ultrasound to evaluate the thyroid bed, central and lateral node compartments should be performed at 6 and 12 months postoperatively, then annually for at least 3 to 5 years for high risk patients (Grade A)

The cervical ultrasound is highly sensitive in the detection of cervical metastases in patients with differentiated thyroid cancer, especially when combined with serum thyroglobulin determinations. ${ }^{88}$ It may occasionally detect metastasis even when TSH-stimulated thyroglobulin is negative. In the Asia-Pacific region, cervical ultrasound plays an ancillary role in the follow up of patients who are at high risk for disease recurrence. ${ }^{10}$ However, this should be performed and interpreted by individuals with significant training and experience in sonographic imaging. 


\section{WORKING GROUP FOR THE REVISED UP - PGH CPG FOR THE MANAGEMENT OF WELL-DIFFERENTIATED THYROID CANCERS IN ADULT FILIPINO PATIENTS}

\author{
Section of Endocrinology \\ Mary Anne Lim-Abrahan, MD \\ Elizabeth Paz-Pacheco, MD \\ Frances Lina Lantion-Ang, MD \\ Laura Trajano-Acampado, MD \\ Myrna Buenaluz-Sedurante, MD \\ Gabriel Jasul, Jr., MD \\ Cecilia Jimeno, MD \\ Iris Thiele Isip-Tan, MD \\ Cecille Añonuevo-Cruz, MD \\ Cherrie Mae Sison, MD \\ Mark Sandoval, MD
}

\author{
Department of \\ Otorhinolaryngology \\ Alfredo Pontejos, Jr., MD \\ Jeannette Matsuo, MD \\ Christine Arquiza, MD \\ Arsenio Cabungcal, MD
}

\author{
Department of Surgery \\ Nelson Cabaluna, MD \\ Arturo dela Pena, MD \\ Daniel dela Paz, Jr., MD \\ Rey Joson, MD \\ Rodney Dofitas, MD \\ Orlino Bisquera, Jr., MD \\ Neresito Espiritu, MD \\ Mark Kho, MD \\ Gemma Leonora Uy, MD
}

\section{Section of Nuclear Medicine \\ Jerry Obaldo, MD \\ Ruben Ogbac, MD}

\section{References}

1. PGH Working Group on Thyroid Cancer. Clinical Practice Guidelines of the Philippine General Hospital for the Management of Thyroid Nodules and Well-differentiated Thyroid Carcinoma. The National Health Science Journal. 2008; 42(1):56-67.

2. Nixon IJ, et al. Thyroid lobectomy for treatment of well differentiated intrathyroid malignancy. http://dx.doi.org/10.1016/j.surg.2011.08.016. Accessed November 2011.

3. Mazzaferri EL and Young RL. Papillary thyroid carcinoma: A 10 year follow-up report of the impact of therapy in 576 patients. Am J Med. 1981; 70:511-518.

4. DeGroot LJ, et al. Natural history, treatment, and course of papillary thyroid carcinoma. J Clin Endocrinol Metab. 1990; 71:414-424.

5. Samaan NA, et al. The results of various modalities of treatment of well differentiated thyroid carcinomas: A retrospective review of 1599 patients. J Clin Endocrinol Metab. 1992; 75:714-720.

6. Hay ID, et al. Papillary thyroid carcinoma managed at the Mayo Clinic during six decades $(1940$ - 1999): Temporal trends in initial therapy and long-term outcome in 2444 consecutively treated patients. Word J Surg. 2002; 26:879-885.

7. Shaha AR, et al. Differentiated thyroid cancer presenting initially with distant metastasis. Am J Surg. 1997; 174:474-476.

8. Sanders LE and Cady B. Differentiated thyroid cancer: Reexamination of risk groups and outcome of treatment. Arch Surg. 1998; 133:419425.

9. Bilimoria KY, Bentrem DJ, Ko CY, et al. Extent of surgery affects survival for papillary thyroid cancer. Ann Surg. 2007; 246:375-381.

10. Sundram F, et al. Well-Differentiated Epithelial Thyroid Cancer Management in the Asia Pacific Region: A Report and Clinical Practice Guideline. Thyroid. November 2006; 16(5):461-469.

11. Racho, Veronica and Lim-Abrahan, Mary Anne. Comparison of Nontotal thyroidectomy versus total thyroidectomy and radioactive iodine ablation for stage 1 and II Papillary Cancer of the Thyroid. Phil J. Internal Medicine. 1998. 36:137-142.

12. Nam-Goong IS, Kim HY, Gong G, et al. Ultrasonography-guided fineneedle aspiration of thyroid incidentaloma: Correlation with pathological findings. Clin Endocrinol (Oxf ). 2004; 60:21-28.

13. Grebe SK, Hay ID. Thyroid cancer nodal metastases: Biologic significance and therapeutic considerations. Surg Oncol Clin N Am. 1996; 5:43-63.

14. Scheumann GF, Gimm O, Wegener G, Hundeshagen H, Dralle H. Prognostic significance and surgical management of locoregional lymph node metastases in papillary thyroid cancer. World J Surg. 1994; 18:559-568.

15. Ito $Y$, Uruno $T$, Nakano $K$, et al. An observation trial without surgical treatment in patients with papillary microcarcinoma of the thyroid. Thyroid. 2003; 13:381-387.
16. Chow SM, Law SC, Chan JK, Au SK, Yau S, Lau WH. Papillary microcarcinoma of the thyroid - Prognostic significance of lymph node metastasis and multifocality. Cancer. 2003; 98:31-40.

17. Hay ID, Grant CS, van Heerden JA, Goellner JR, Ebersold JR, Bergstralh EJ. Papillary thyroid microcarcinoma: A study of 535 cases observed in a 50-year period. Surgery. 1992; 112:1139-1146; discussion 1146-1147.

18. Solorzano CC, Carneiro DM, Ramirez M, Lee TM, Irvin GL3rd Surgeon-performed ultrasound in the management of thyroid malignancy. Am Surg. 2004; 70:576-580; discussion 580-582.

19. Shimamoto K, Satake H, Sawaki A, Ishigaki T, Funahashi H, Imai T. Preoperative staging of thyroid papillary carcinoma with ultrasonography. Eur J Radiol. 1998; 29:4-10.

20. Stulak JM, Grant CS, Farley DR, et al. Value of preoperative ultrasonography in the surgical management of initial and reoperative papillary thyroid cancer. Arch Surg. 2006; 141:489-494.

21. Kouvaraki MA, Shapiro SE, Fornage BD, et al. Role of preoperative ultrasonography in the surgical management of patients with thyroid cancer. Surgery. 2003; 134:946-954; discussion 954-955.

22. Cooper DS, et al. Revised American Thyroid Association Management Guidelines for Patients with Thyroid Nodules and Differentiated Thyroid Cancer. Thyroid. 2009; 19(11):1167-1217.

23. Mulcahy MM, et al. Relative Accuracy of Fine-Needle Aspiration and Frozen Section in the Diagnosis of Well-Differentiated Thyroid Cancer. The Laryngoscope. 1998; 108(4):494-496.

24. Huber GF, et al. Intraoperative frozen-section analysis for thyroid nodules: a step toward clarity or confusion? Arch Otolaryngol Head Neck Surg. 2007; 133(9):874-81.

25. Lumachi F, et al. FNA cytology and frozen section examination in patients with follicular lesions of the thyroid gland. Anticancer Res. 2009; 29(12):5255-7.

26. Podnos YD, Smith D, Wagman LD, Ellenhorn JD. The implication of lymph node metastasis on survival in patients with welldifferentiated thyroid cancer. Am Surg. 2005; 71:731-734.

27. Zaydfudim V, Feurer ID, Griffin MR, Phay JE. The impact of lymph node involvement on survival in patients with papillary and follicular thyroid carcinoma. Surgery. 2008; 144:1070-1077; discussion 1077-1078.

28. Leboulleux S, Rubino C, Baudin E, et al. Prognostic factors for persistent or recurrent disease of papillary thyroid carcinoma with neck lymph node metastases and/or tumor extension beyond the thyroid capsule at initial diagnosis. J Clin Endocrinol Metab. 2005; 90:5723-5729.

29. Tissel LE, et al. Improved survival of patients with papillary thyroid cancer after surgical microdissection. World J Surg. 1996; 20:854-859.

30. Scheumann GF, et al. Prognostic significance and surgical management of locoregional lymph node metastasis in papillary thyroid cancer. World J Surg. 1994; 18:559-568. 
31. Olson JA Jr, et al. Parathyroid autotransplantation during thyroidectomy. Results of long-term follow up. Ann Surg. 1996; 223:472-478.

32. Gimm $\mathrm{O}$, et al. Pattern of lymph node metastases in papillary thyroid carcinoma. Br J Surg. 1998; 85:252-254.

33. Henry JF, et al. Morbidity of prophylactic lymph node dissection in the central neck area in patients with papillary thyroid carcinoma. Langenbecks Arch Surg. 1998; 383:167-169.

34. Cheah WK, et al. Complications of neck dissection for thyroid cancer. World J Surg. 2002; 26:1013-1016.

35. Pacini F, Elisei R, Capezzone M, et al. Contralateral papillary thyroid cancer is frequent at completion thyroidectomy with no difference in low- and high- risk patients. Thyroid. 2001; 11:877-881.

36. Erdem E, et al. Comparision of completion thyroidectomy and primary surgery for differentiated thyroid carcinoma. Eur J Surg Oncol. 2003; 29:747-749.

37. Shaha AR, et al. Completion thyroidectomy: A critical appraisal. Surgery. 1992; 112:1148-1153.

38. Sherman SI, et al. Prospective multicenter study of thyroid carcinoma treatment: Initial analysis of staging and outcome. National Thyroid Cancer Treatment Cooperative Study Registry Group. Cancer. 1998; 83:1012-1021

39. Shah MD, et al. Clinical course of thyroid carcinoma after neck dissection. Laryngoscope. 2003; 113:2102-2107.

40. Mazzaferri EL and Jhiang SM. Long-term impact of initial surgical and medical therapy on papillary and follicular thyroid cancer. Am J Med. 1994; 97:418-428.

41. Mazzaferri EL and Jhiang SM. Differentiated thyroid cancer longterm impact of initial therapy. Trans Am Clin Climatol Assoc. 1994; 106:151-168.

42. Sawka AM, et al. A systematic review and metaanalysis of the effectiveness of radioactive iodine remnant ablation for welldifferentiated thyroid cancer. J Clin Endocrinol Metab. 2004; 89:36683676.

43. Mazzaferri EL. Thyroid remnant 131I ablation for papillary and follicular thyroid carcinoma. Thyroid. 1997; 7:265-271.

44. Carril JM, et al. Total body scintigraphy with thallium-201 and iodine-131 in the follow up differentiated thyroid cancer. J Nucl Med. 1997; 38:686-692.

45. Muratet JP, et al. Predicting the efficacy of first iodine-131 treatment in differentiated thyroid cancer. J Nucl Med. 1997; 38:1362-1368.

46. Park HM, et al. Detection of thyroid remnant/metastasis without stunning: An ongoing dilemma. Thyroid. 1997; 7:277-280.

47. Hilditch TE, et al. Self-stunning in thyroid ablation: Evidence from comparative studies of diagnostic 131I and 123I. Eur J Nucl Med Mol Imaging. 2002; 29:783-788.

48. Edmonds CJ, Hayes S, Kermode JC, Thompson BD. Measurement of serum TSH and thyroid hormones in the management of treatment of thyroid carcinoma with radioiodine. Br J Radiol. 1977; 50:799-807.

49. Liel Y. Preparation for radioactive iodine administration in differentiated thyroid cancer patients. Clin Endocrinol (Oxf ). 2002; 57:523-527.

50. Sanchez R, Espinosa-de-los-Monteros AL, Mendoza V, et al. Adequate thyroid- stimulating hormone levels after levothyroxine discontinuation in the follow-up of patients with well-differentiated thyroid carcinoma. Arch Med Res. 2002; 33:478-481.

51. Grigsby PW, Siegel BA, Bekker S, Clutter WE, Moley JF. Preparation of patients with thyroid cancer for 131I scintigraphy or therapy by $1-$ 3 weeks of thyroxine discontinuation. J Nucl Med. 2004; 45:567-570.

52. Serhal DI, Nasrallah MP, Arafah BM. Rapid rise in serum thyrotropin concentrations after thyroidectomy or withdrawal of suppressive thyroxine therapy in preparation for radioactive iodine administration to patients with differentiated thyroid cancer. J Clin Endocrinol Metab. 2004; 89:3285-3289.

53. Pacini F, Ladenson PW, Schlumberger M, et al. Radioiodine ablation of thyroid remnants after preparation with recombinant human thyrotropin in differentiated thyroid carcinoma: Results of an international, randomized, controlled study. J Clin Endocrinol Metab. 2006; 91:926-932.

54. Pilli T, Brianzoni E, Capoccetti F, et al. A comparison of 1850 (50 mCi) and $3700 \mathrm{MBq}(100 \mathrm{mCi})$ 131-iodine administered doses for recombinant thyrotropin-stimulated postoperative thyroid remnant ablation in differentiated thyroid cancer. J Clin Endocrinol Metab. 2007; 92:3542-3546.
55. Chianelli M, Todino V, Graziano F, et al. Low dose (2.0 GBq; $54 \mathrm{mCi})$ radioiodine postsurgical remnant ablation in thyroid cancer: comparison between hormone withdrawal and use of rhTSH in low risk patients. Eur J Endocrinol. 2009; 160: 431-436.

56. Tuttle RM, Brokhin M, Omry G, et al. Recombinant human TSHassisted radioactive iodine remnant ablation achieves short-term clinical recurrence rates similar to those of traditional thyroid hormone withdrawal. J Nucl Med. 2008; 49:764-770.

57. Rosario PW, et al. Efficacy of low and high 131I doses for thyroid remnant ablation in patients with differentiated thyroid carcinoma based on post-operative cervical uptake. Nucl Med Commun. 2004; 25:1077-1081.

58. Bal C, et al. Prospective randomized clinical trial to evaluate the optimal dose of 131 I for remnant ablation in patients with differentiated thyroid carcinoma. Cancer. 1996; 77:2574-2580.

59. Creutzig H. High or low dose radioiodine ablation of thyroid remnants? Eur J Nucl Med. 1987; 12:500-502.

60. Johansen K, et al. Comparison of $1073 \mathrm{MBq}$ and $3700 \mathrm{MBq}$ iodine-131 in postoperative ablation of residual thyroid tissue in patients with differentiated thyroid cancer. J Nucl Med. 1991; 32:252-254.

61. Doi SA, et al. Ablation of the thyroid remnant and 131I dose in differentiated thyroid cancer. Clin Endocrinol (Oxf). 2000; 52:765-773.

62. McGriff NJ, et al. Effects of thyroid hormone suppression therapy on adverse clinical outcomes in thyroid cancer. Ann Med. 2002; 34:554564.

63. Pujol P, et al. Degree of thyrotropin suppression as a prognostic determinant in differentiated thyroid cancer. J Clin Endocrinol Metab. 1996; 81:4318-4323.

64. Cooper DS, et al. Thyrotropin suppression and disease progression in patients with differentiated thyroid cancer: Results from the National Thyroid Cancer Treatment Cooperative Registry. Thyroid. 1998; 8:737-744.

65. Surks MI, et al. Subclinical thyroid disease: Scientific review and guidelines for diagnosis and management. JAMA. 2004; 291(2):228238.

66. Tenerz A, et al. Is a more active attitude warranted in patients with subclinical thyrotoxicosis? J Intern Med. 1990; 228:229-233.

67. Sawin CT, et al. Low serum thyrotropin concentrations as a risk factor for atrial fibrillation in older patients. N Engl J Med. 1994; 331:12491252.

68. Wilson PC, et al. The management of advanced thyroid cancer. Clin Oncol (R Coll Radiol). 2004; 16:561-568.

69. Goettlieb JA, et al. Chemotherapy of thyroid cancer. An evaluation of experience with 37 patients. Cancer. 1992; 30:848-853.

70. Goettlieb JA, et al. Chemotherapy of thyroid cancer with adriamycin N Engl J Med. 1974; 290:93-197.

71. O'Bryan RM, et al. Dose response evaluation of adriamycin in human neoplasia. Cancer. 1977; 39:1940-1948.

72. Pacini F, et al. Treatment of refractory thyroid cancer with adriamycin. Drugs Experimental Clin Res. 1984; 10:911-916.

73. Haugen BR. Management of the patient with progressive radioiodine non-responsive disease. Semin Surg Oncol. 1999; 16:34-41.

74. Thyroid Carcinoma Task Force. AACE/AAES Medical/Surgical guidelines for clinical practice: Management of thyroid carcinoma. Endocrine Practice. May-June 2001; 7(3): 203.

75. Mazzaferri, EL and Kloos, RT. Current approaches to primary therapy for papillary and fFollicular thyroid carcinoma. J Clin Endocrinol Metab. 2001; 84(4):1447.

76. Sherman, Steven I. Radioiodine treatment of differentiated thyroid cancer. http://www.uptodate.com. Version 13.1, last revised January 14, 2001. Accessed September 2011.

77. Edmonds CJ, et al. Measurement of serum TSH and thyroid hormones in the management of treatment of thyroid carcinoma with radioiodine. British Journal of Radiology. November 1977; 50(599):799-807.

78. Toubeau M, et al. Predictive value for disease progression of serum thyroglobulin levels measured in the postoperative period and after (131)I ablation therapy in patients with differentiated thyroid cancer. J Nucl Med. 2004; 45:988-994.

79. Rouxel A, et al. Prognostic factors associated with the survival of patients developing loco-regional recurrences of differentiated thyroid carcinomas. J Clin Endocrinol Metab. 2004; 89:5362-5368.

80. Spencer CA, et al. Detection of residual and recurrent differentiated thyroid carcinoma by serum thyroglobulin measurement. Thyroid. $1999 ; 9: 435-441$. 
81. Spencer CA. Challenges of serum thyroglobulin measurement in the presence of thyroglobulin autoantibodies. J Clin Endocrinol Metab 2004; 89:3702-3704.

82. Bachelot A, et al. Neck recurrence from thyroid carcinoma: Serum thyroglobulin and high-dose total body scan are not reliable criteria for cure after radioiodine treatment. Clin Endocrinol (Oxf). 2005; 62:376-379.

83. Baudin E, et al. Positive predictive value of serum thyroglobulin levels, measured during the first year of follow-up after thyroid hormone withdrawal, in thyroid cancer patients. J Clin Endocrinol Metab. 2003; 88:1107-1111.

84. Schaap J, et al. Does radioiodine therapy have disadvantageous effects in non-iodine accumulating differentiated thyroid carcinoma? Clin Endocrinol. 2002; 57:117-124.

85. Spencer CA, et al. Serum thyroglobulin antibodies: Prevalence, influence on serum thyroglobulin measurement, and prognostic significance in patients with differentiated thyroid carcinoma. J Clin Endocrinol Metab 1998; 83:1121-1127.

86. Chiovato L, et al. Disappearance of humoral thyroid autoimmunity after complete removal of thyroid antigens. Ann Intern Med. 2003; 139:346-351.

87. Rubello D, Casara D, Girelli ME, Piccolo M, Busnardo B. Clinical meaning of circulating antithyroglobulin antibodies in differentiated thyroid cancer: A prospective study. J Nucl Med. 1992; 33:1478-80
88. Pacini F, et al. Recombinant human thyrotropin-stimulated serum thyroglobulin combined with neck ultrasonography has the highest sensitivity in monitoring differentiated thyroid carcinoma. J Clin Endocrinol Metab. 2003; 88:3668-3673.

89. Mazzaferri EL, et al. Author's response: A consensus report of the role of serum thyroglobulin as a monitoring method for low-risk patients with papillary thyroid cancer. J Clin Endocrinol Metab. 2003; 88:4508-4509.

90. Schlumberger $\mathrm{M}$, et al. 131-I Therapy for elevated thyroglobulin levels. Thyroid. 1997; 7:273-276.

91. Mazzaferri EL, et al. Current approaches to primary therapy for papillary and follicular thyroid cancer. J Clin Endocrinol Metab. 2001 86:1447-1463.

92. Pacini F, et al. Diagnostic 131-iodine whole-body scan may be avoided in thyroid cancer patients who have undetectable stimulated serum thyroglobulin levels after initial treatment. J Clin Endocrinol Metab. 2002; 87:1499-1501.

93. Koh JM, et al. Effects of therapeutic doses of $131 \mathrm{I}$ in thyroid papillary carcinoma patients with elevated thyroglobulin level and negative 131I whole-body scan: Comparative study. Clin Endocrinol (Oxf). 2003; 58:421-427.

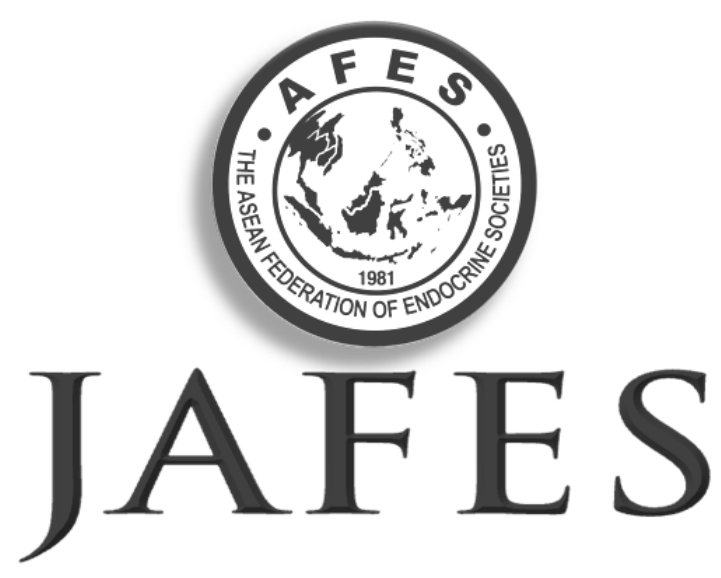

\section{Topics with cultural interest $=$ Images of Asia at JAFES aAsia.com}

